port was located left at $8 \mathrm{~cm}$ from umbilicus, second port was 20 $\mathrm{mm}$ sized at umbilicus, third port was located right at $8 \mathrm{~cm}$ from umbilicus, and fourth was located right at $8 \mathrm{~cm}$ from the third port (near the right flank). Uterus was tied with needle-straightened multifilament Vicryl 2-0 and tagged uterus was manipulated by fourth arm of the robot. If additional traction is required, instrument was inserted though the umbilical trocar site. During operation, the tagged uterus was successfully manipulated and appropriate parametrial space was exposed. Pathologically, all surgical margins were not involved with cancer. No tumor cells were seen in cytologic exam before and after the colpotomy. Robotic radical hysterectomy can be easily and safely done with the traction of tagged uterine suture.

Surgical Film (F4)

Surgical Techniques \& Perioperative Management

https://doi.org/10.3802/jgo.2021.32.S1.F4

\section{Vaginal assisted laparoscopic radical hysterectomy (nerve sparing) after LACC era}

\section{Supachai Raungkaewmanee, Uraiwan Khomphaiboonkij, ” Supakorn Pitakkarnkul, Panwad Ratanasrithong, Warangkana Kolaka}

National Cancer Institute Thailand, Bangkok, Thailand (awan1985@hotmail.com)

From Laparoscopic Approach to Cervical Cancer (LACC) trial, patients who underwent minimally invasive radical hysterectomy for early-stage cervical cancer had lower rates of disease-free survival and overall survival and a higher rate of locoregional recurrence. There are potential reasons for the inferior oncologic outcomes, that the routine usage of a uterine manipulator and intracorporeal colpotomy might increase the propensity for tumor spillage, effect of the insufflation gas $\left(\mathrm{CO}_{2}\right)$ on tumor-cell growth or spread. We use selection criteria and change some steps of procedure to reduce risk of recurrent disease, such as: 1) tumor size $<2 \mathrm{~cm}, 2$ ) no lymph-vascular space invasion, 3) not using uterine manipulator, 4) transvaginal colpotomy, and 5) closing vaginal cuff transvaginally. Steps of procedure divided into laparoscopic and vaginal parts. Laparoscopic part steps are as follows: 1) after starting pneumoperitoneum with $\mathrm{CO}_{2}$ pressure $10-12 \mathrm{mmHg}$, abdominal exploration is performed; 2) bilateral pelvic lymphadenectomy is performed then frozen section; 3) creating paravesical and pararectal space then identifing hypogastric nerve; 4) radical hysterectomy performed as conventional; 5) cutting uterine branch of hypogastric nerve then dissecting laterally; and 6) suturing to mark vaginal wall. Vaginal part follows as: 1) identifing suture marker; 2) opening vaginal cuff by scalpel; 3) removing specimen then painting with povidone solution; and 4) closing vaginal stump transvaginally. The advantages of minimal invasive surgery should not be abandoned because of the unexpected findings of the LACC trial. We continue to be critically assessed by discussion with patients before surgery. Ongoing and future randomized controlled trials are needed and should be strongly supported.

Surgical Film (F5)

Cervical Cancer

https://doi.org/10.3802/jgo.2021.32.S1.F5

\section{Robotic assisted radical vaginal trachelectomy (fertility sparing surgery)}

\author{
Amany Abdelwadoud Makroum, ${ }^{1, *}$ Jung-Yun Lee ${ }^{2}$ \\ 'Department of Obstetrics and Gynecology, Faculty of Medicine, \\ Mansoura University, Mansoura, Egypt (amany_makroum@mans.edu.eg) \\ 2Department of Obstetrics and Gynecology, Institute of Women's Life Medical \\ Science, Yonsei University College of Medicine, Seoul, Korea
}

A 32-year-old nulliparous woman with early-stage invasive cervical adenocarcinoma underwent robotic assisted radical vaginal trachelectomy (fertility sparing surgery). Steps were followed as: 1) Sentinel lymph node biopsy, 2) Paravesical and Pararectal spaces dissection, 3) Ureter \& uterine artery identification, 4) Division of anterior vesico-uterine ligaments (bladder pillars), 5) Recto-vaginal space dissection (uterosacral ligaments division), 6) Colpotomy, 7) Cervical amputation, 8) Frozen biopsy, 9) Cervical cerclage, and 10) Utero-vaginal anastomosis.

Surgical Film (F6)

Uterine Sarcoma

https://doi.org/10.3802/jgo.2021.32.S1.F6

\section{vNOTE hysterectomy for adenosarcoma in patient with complicated abdominal surgical history}

\author{
Thanasak Sueblinvong, " Teresa Walsh \\ Kaiser Permanente, Oakland, CA, USA (Thanasak.Sueblinvong@kp.org)
}

This is a 72-year-old with postmenopausal bleeding underwent a hysteroscopic dilation and curettage which revealed adenosarcoma. Her underlying medical problems included chronic kidney disease (glomerular filtration rate 19); obesity (body mass index 38) with associated comorbidities; also an abdominal aortic aneurysm (AAA) with a diameter $7.4 \mathrm{~cm}$. Due to newly diagnosis of cancer and vaginal bleeding, her AAA repair process was put on hold. She had a complicated surgical history. In 2016, she had large bowel obstruction due to underlying 